\title{
A TRULY AUTONOMOUS ROBOTIC SYSTEM THROUGH SELF-MAINTAIED ENERGY
}

\author{
Trung Dung Ngo \\ Center for Embedded Software Systems (CISS) \\ Aalborg University \\ Fr. Bajersvej 7B, 9220 Aalborg East \\ dungnt@cs.aau.dk
}

\author{
Henrik Schiøler \\ Control Department \\ Aalborg University \\ Fr. Bajersvej 7B, 9220 Aalborg East \\ henrik@control.aau.dk
}

\begin{abstract}
World research in autonomous robots has focused on behaviors inspired by natural animal such as flocking, foraging, collecting, and sharing of ants, birds, and insects. The word "autonomous" implies that robots should absolutely behave without human intervention. However, robots are not truly autonomous if it can find food to survive. Autonomous mobile robots are usually equipped with a finite amount of energy, it can thus operate in a finite time. To solve the limit, this paper issues an approach to truly autonomous robots through capability of self-maintaining energy, which is dominantly inspired from swarm behavior of collecting and sharing food of honey-bee. We describe practical deployment of a group of mobile robots, named CISSbots, that are capable of carrying and exchanging fuel, e.g. battery to other robot. The battery exchange algorithm basically based on probabilistic modeling of total energy on each robot located in local vicinity is then described. Subsequently, modular hardware and control architecture of such robots is presented. Experimental scenarios are addtionally illustrated as demonstrations of the approach.
\end{abstract}

Keywords: Truly Autonomous, Sociable Robots, Probabilistic Energy, Neighbourhood.

\section{INTRODUCTION}

Autonomous robot is originally initialized from research on natural animal behaviors. Since individuals live in a society, they always keep relationship and social interaction, create group in specific norm, and adhere the convention. Concerning truly autonomous mobile robots, we are expecting to create a society of mobile robots where robots itself should find a food and share it with other robots. Furthermore, such robots in the society are capable of mutually sharing not only food, but also task, information, recognition and even intelligence to other robots. Here, we create a new approach to fairly efficient energy sharing for group of mobile robots that are all constructed in the same architectural morphology and control system. Thus every robot has also fairly same task of carrying battery and exchanging to other robots. Further such robots can additionally be assigned other different tasks depending on the environment and mission. The establishment of energy exchange among distributed robots is referred to probabilistic model in which dynamical variables are remaining energy of robots, related distances between them, absolute distance to closest full charged battery station, work-load of assigned task, and history. On the horizon, we are establishing a fully distributed autonomous mobile robot system with long-lived property conducted by probabilistic distribution among robots.

In the paper, we issue a new prototype of mobile robots that are capable of fairly carrying battery and exchanging to other robots. We propose an algorithm of self-distributed energy based on probabilistic model. Early results of control hardware architecture are also presented. Finally, we show experimental deployment of our mobile robots.

\section{RELATED WORKS}

Concerning law of conservation of energy, energy can not created and destroyed, but it can change its form. With mobile robots, it must be necessary to be equipped with a tank of energy to power its operation and then the tank must be refueled again. Like transporting vehicles, it can be equipped a tank of energy e.g. gas, petrol, but the most of electric vehicles use rechargeable battery packs. Other robot is attached a solar cell or wind cell on the top to salvage natural sources. In fact, we know that a mobile robot will have life-span of no longer than finite time limited by available energy. Therefore, to create long-lived mobile robot, the volume of fuel tank should be increased, the fuel material should be more concentrated or natural condition of sunlight or wind should be stable time by time. However, criteria all are impossibly fulfilled due to real limitation of tank material, size, fuel concentration, natural condition, and operation environment.

In another classical method of energy conversion, European researchers have investigated collecting and digesting food of natural animals, e.g. sugar, pistil, flies and a digestive mechanism of food to transfer naturally collected food to energy for mobile robot. Ecobot I [1, 13] with a sugar digestive mechanism demonstrates possibility of the biological method. Further, Ecobot II can catch home flies, and then digests it before their exoskeletons are turned into electricity. But, sugar or flies are unavailable sources.

Another approach to long-lived mobile robot is recharging stations. The approach is widely applied for vacuum cleaning robot that autonomously moves around to clean up and return to docking station to be recharged in 
indoor environment. Normally, such robot uses rechargeable batteries as power sources, and of course need to be charged again for a few hours. The typical method to guide robot to reach docking is use of proximity sensor and communication to robots and docking. Silverman [7] and Seungjun Oh [8] describe their implementation in which autonomous recharging docking mechanism is specially designed with infrared proximity and laser range sensors to guide robot to go back and firmly connect with the station. The technique is slightly borrowed by landing technique of airplane. Moreover, Hada and Yuta at el. [9] give results of the week-long repetitive docking experiment. Their robot is equipped infrared sensor and reflective tape on the floor to guide robot to docking station. But the most difficulty of such technique to successfully implement is path planning that enables robot to contact charging station precisely. In the case, particle filtering [15] or Kalman filtering algorithm has been usually applied to estimate a suitable path. As an extension, multi-charging stations to maximize longevity of distributed robotic teams is considered in some practical experiments. Nevertheless, the solution is really satisfied to simple autonomous robot with low-level mission and no high demand of charging time, and traveling time from target to charging station.

Another approach to prolong longevity of mobile robot's power source is efficient use of a finite energy amount available. To save total energy of mobile robot, optimizing integrated devices on the robot and its motion planning to reduce operation power is world-widely researched. Conventionally, hardware configuration of mobile robots obtains embedded electronic boards, several actuator and sensors. Because the electronic devices always consume a finite energy even it is not necessary to be used at a time, the devices should be turned off or switched to standby mode to save overall energy. For instance, Barili et al. [16] considers the concept of controlling velocities of DC-motors to save energy. But the technique is only to save overall power for mobile robot and then prolonging longevity of robot in limited amount of energy, it is not sufficiently capable of extending or changing the total energy to prolong longevity of mobile robots.

Nearby, Zerowski and Vaughan et al. [12] propose an interesting approach: a tanker robot that is specially used as "mother" robot. The robot traverses to record the temporal position of "worker" robot and then distribute energical cells to "worker" robots if demanded. This proposal is compared with daily work of gas trucks that usually deliver gas from main repository to local gas station or tanker aircraft to fighter aircraft on the sky. Advantages of the proposal are cheaper and simpler complexity of "worker" robot and efficient energy of systems. But drawbacks of specializing tasks of robots can easily manifest: single-task on each robot is assigned and traveling distance of tanker will be too long to workers not close to fuel station. Thus it is impossible for emergence case when worker is too far away charging station needs battery. Additionally, current results are presented in simulation, not practical achievement. They did not also show how to implement such a described robotic system.

\section{ALGORITHM OF SELF-MAINTAINED ENERGY}

The energy consumption of the CISSbot is approximately calculated by accumulating energy consumption of electronic boards, battery exchange agents and essential amount of two wheeled DC motors. Based on property of CISSbot and measurement of energy consumption of electronic elements, we present a probabilistic model for propagation of battery resources through battery exchange. Initially we synthesize remaining energy capacity to decide next status of robot. Then, we propose methods to engage in battery exchange in a distributed robotic system in which each robot has to negotiate with other robot around it to exchange energy. In this paper, we focus on centralized algorithm of battery exchange that is executed on host. Information of remaining battery, position, and task of CISSbots is managed on the host via wireless communication.

We assume that at a time $t$ the robot $r_{i}$ has remaining energy capability $E_{i}$ that is collected from individual energy $e_{j}$ of battery available in eight holders nob. Therefore, we can synthesize the total energy of robot $r_{i}$ on probabilistic number of batteries at a time:

$$
E_{i}=\sum_{i=1}^{n o b} e_{i}
$$

Also at time $t$, there exist $k$ robots in the robot $r_{i}$ 's vicinity with corresponding distance $d_{k i}$ and itself is distance $d_{i s}$ far from a closest charging station. We define $C_{i}(t)$ as average energy consumption of the robot $r_{i}$ freely moving in a unit of distance without other tasks. Thus, there exists two possibilities for robot $r_{i}$ to be fueled: robot $r_{i}$ consumes amount of $d_{i s} * C_{i}(t)$ if it wishes to go back to the charging station to take newly charged batteries, and other robots $r_{i}\{i: 1 \ldots \mathrm{k}, \mathrm{i} \neq \mathrm{k}\}$ consume energy $d_{k i} * C_{i}(t)$ if they wish to go the current position of robot $r_{i}$ to exchange batteries.

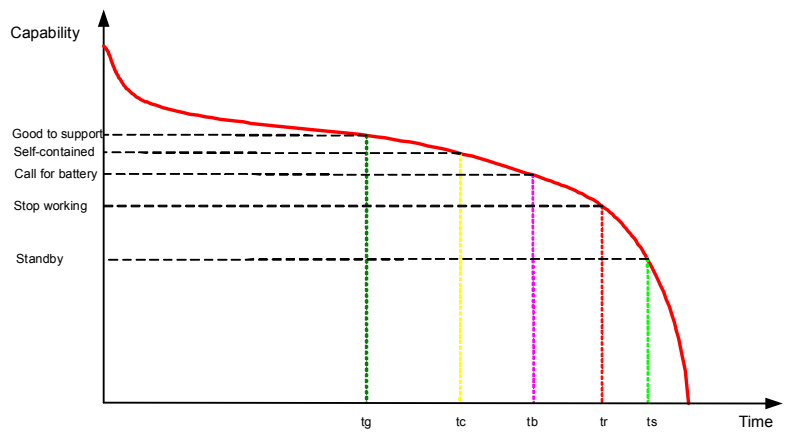

Fig.1. Battery status to support decision for CISSbot 
We assume that $t+\Delta t$ is time when robot $r_{i}$ is already recharged. The total energy of robot $r_{i}$ at time $t+\Delta t$ is substitution of remaining energy at time $t$ and energy consumed on traveling distance $d_{i s}$ and $d_{k i}$. At time $t+\Delta t$, remaining energy of robots around the robot $r_{i}$ is estimated:

$$
E_{i}(t+\Delta t)=E_{i}(t)-d_{i s} * C_{i}(t)
$$

CISSbots itself always checks battery status and keep communicating with the host. Assuming that a robot $r_{i}$ needs to be charged, indicated by battery management system, it will send a call_for_battery to the host. The host will search closest charging station and the other robot with high energy around $r_{i}$ to order which robot is going to go and exchange battery with $r_{i}$ or command $r_{i}$ to go back the closest charging station. Thereby, we can formulate energy distribution in term of algorithm of comparison and negotiation of remaining energy of robots to issue the decision of battery exchange. We issue probabilistic algorithm of battery distribution for CISSbot, including two procedures: searching for closest charging station and robots in robot $r_{i}$ 's vicinity with corresponding distances and remaining energy; and decision:

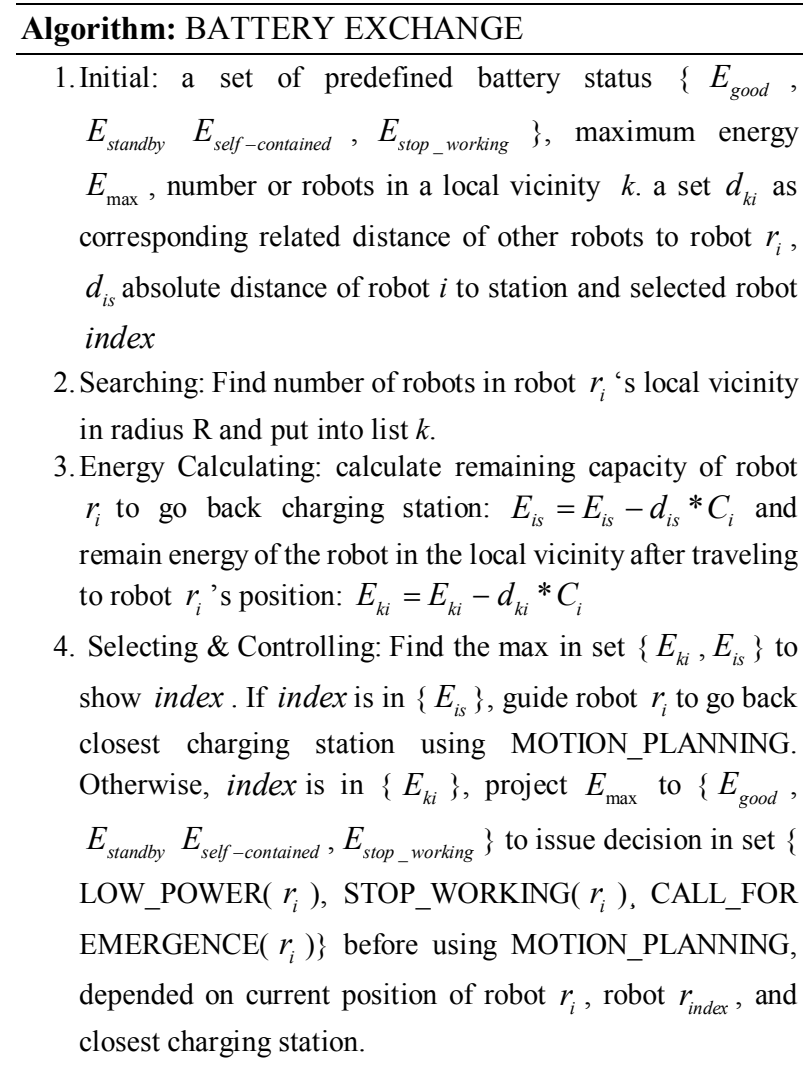

\section{CISSBOT ARCHITECTURE}

4.1 Mechanical prototype
In the project we have designed a standard mobile robot architecture constructed by two-wheel differential steerings with two additional points of contact. Therefore, kinematic motion of the robot is only depending on kinematics sliding constraints of standard two-wheels.

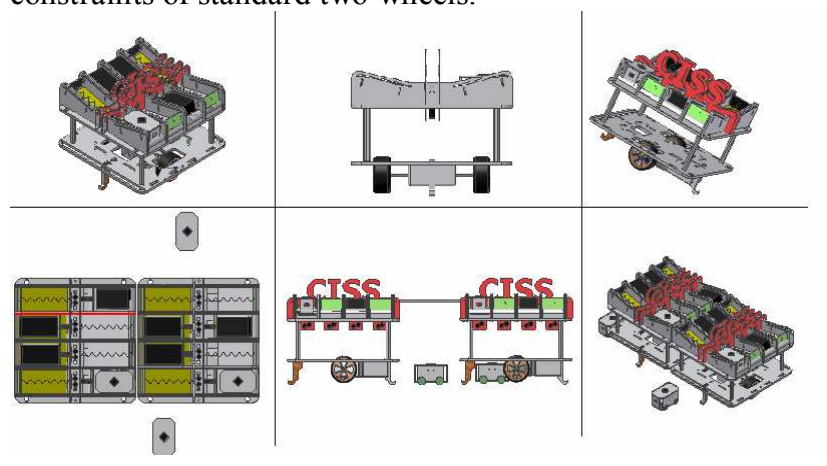

Fig.2 CAD model of CISSbot

The robot is architected in two open-layers in which the lower layer contains central control system and upper layer is specially built for battery exchange. With the open-mechanical architecture, the robot can be extended with other layer based on assigned mission. For example, a manipulator is possibly attached on the highest layer of the robot to be able to scan and pick up specified objects on the floor. Since challenges in mechatronics design are approaching to integration of both mechanical and embedded electronics, we firstly illustrate overall architecture of mobile robot in CAD model. Consequently, using the standard size of $9 \mathrm{~V}$ rechargeable battery available on the market, the model of the lower layer is definitely designed to fit into motion systems of differential wheels, castors and electronic parts of infrared array, odometer, digital compass radio communication and infrared local communication as well. More functionality, the upper layer is generated with 8 parallel sliding battery holders integrated with miniature linear actuation systems and light indicators. Specially, the linear actuation system is embeddingly created to transfer the rotation force to translation force for pushing battery cells or micro-robots. The layer is additionally implemented with particular hook mechatronic systems for rescue solution. On real mobile robot, all parts of both lower and upper layers are assembled from pieces of acrylic plastic materials, thus it is very easy to modify or change for any requirement as a result. Generally, the CISSbot architecture might partly fulfill basic criteria of flexible mobile robot that are mainly used in laboratory experiments.

\subsection{Electronics}

To control the behavior of CISSbot, electronic circuit boards are suitably designed for assigned task of such robots. As basically required for every mobile robot, the boards consist of integrated elements of central processors, sensing and actuation. In the project, we have chosen a design technique of functionally modular boards that obtain 
specialized function. The electronic boards are connected to form overall architecture laid out between two mechanical layers of the robot: mainboard, battery exchange, battery management, local sensing and communication, wireless communication, digital compass, infrared sensor array, and odometer. The layout of printed circuit boards (PCBs) of such elements can be seen in the figure 2 .

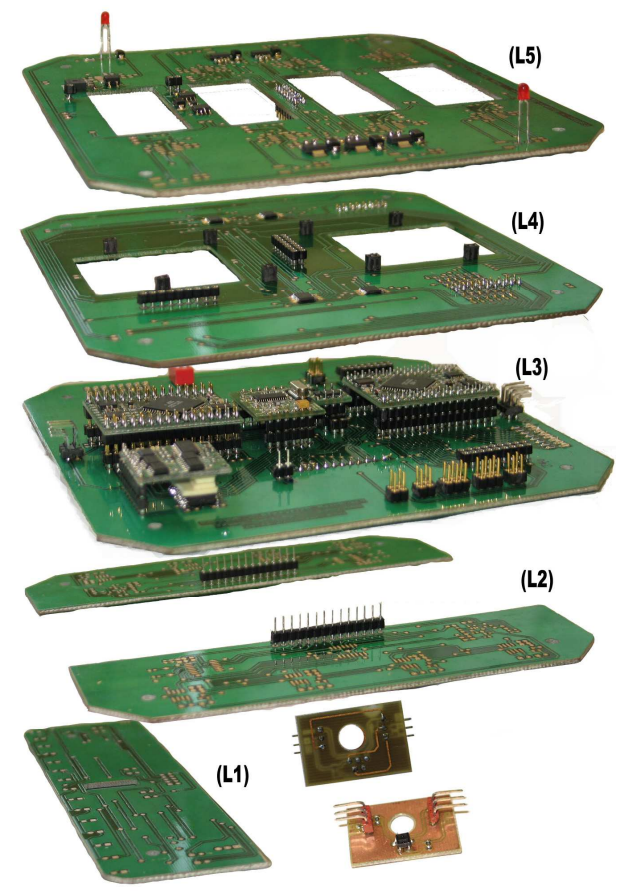

Fig.2. Modular electronics of CISSbot

L1) is the lowest layer laid-out under the bottom mechanical layer of the robot where infrared sensor array works as a vision system to track black or white line on contrast background, alternatively to guide the robot follow the line precisely, and Hamamatsu odometer is designed to estimate speed of the robot.

L2) is the two miniature infrared sensor boards that is flexible design for both local communication between two robots when contacted for battery exchange and proximity sensing for short range measurement.

L3) is the mainboard that control the overall behavior of the robot. On the board, ATMEGA128 adapters are used as main processors that are inter-connected to communicate via $\mathrm{I}^{2} \mathrm{C}$ protocol. Chipcon radio communication adapter is employed to generate global communication among robots as well as host computer. To provide sensing data for localization techniques, Hitachi digital compass is additionally associated with odometer and infrared array. A motor controller is also plugged in on the board to control two differential wheels. Generally, the mainboard is an open-slot board, thus it is easy to expand with extra sensing or actuation modules that will be able to be used in the future to more difficult tasks.
L4) is a battery motor controller board that is created with multiplexer 1 to 16 to reduce number of inputs to control 16 miniature linear actuators. Thus, only one H-bridge motor controller is used to control the whole process of battery exchange, inter-hooks for rescue or chain of mobile robots through encoding commands from main processor.

L5) is a battery management board that is electronical computational circuit that consists of current flow rectifier, DC/DC converter to support a stable voltage for whole electronics parts, light indicator for connected batteries, overall current limiter, multiple voltage regulator outputs for different modules, and sub-circuit of battery measurement. The Maxim chip circuit of battery measurement, which is of major importance must indicate instantaneous values of batteries on each holder in order to decide battery exchange operation on the robot.

\subsection{Controller}

We have built a layered architecture of separately functional modules for our robotic system, which is projected to hardware architecture and software organization. Characteristics of the organization are primarily based on roles of functional modules and their reciprocal relation in the system. The architecture is typically established in the manner of input-processing-output in two levels: low-level processing and high-level processing. In the low-level processing, software drivers are independently implemented for corresponding hardware modules to issue sensing data such as infrared sensing, infrared communication, wireless communication and so forth or to control actuation such as DC motors of wheels, linear motors of battery exchange and so forth.

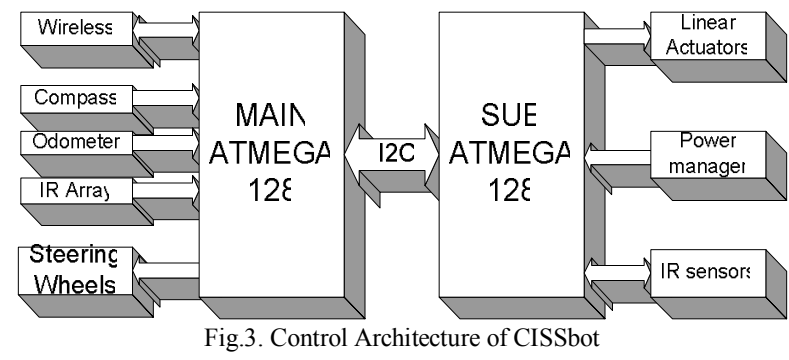

In fact, precision of the system is due to not only sensory data captured by functional sensory modules but also data fusion technique. Here, to increase effect of sensory data, we implemented high-level processing over the low-level processing as a middleware. Point to figure 3 fuses sensory data into data association that is software-implemented in two processors to control robot behaviors. In a view, arrows in figure 3 show the interactive direction between modules with processor. The architecture is divided into two areas of central processing and battery exchange corresponding to mechanical prototype of the CISSbot. The central processing includes global communication among robots as well as interior positioning sensors to localize robot's position while the battery exchange involves battery 
management for each battery holder, and local communication and linear motor system for battery exchanging process. Increasingly, PID controller and Kalman fillering are implemented to control two wheeled DC motor of CISSbots to follow planning path in the middleware.

\section{EXPERMENTAL SCENARIOS}

In the initial scenario we only intend to demonstrate our approach on uniquely probabilistic distributed energy mobile robots towards truly autonomous mobile robots. Thereby, we setup a simple mapping field for CISSbot experiment. The scenario is generated by a 10x10 orthogonal grid of white tapes on the black plastic carpet, shown in figure 4.

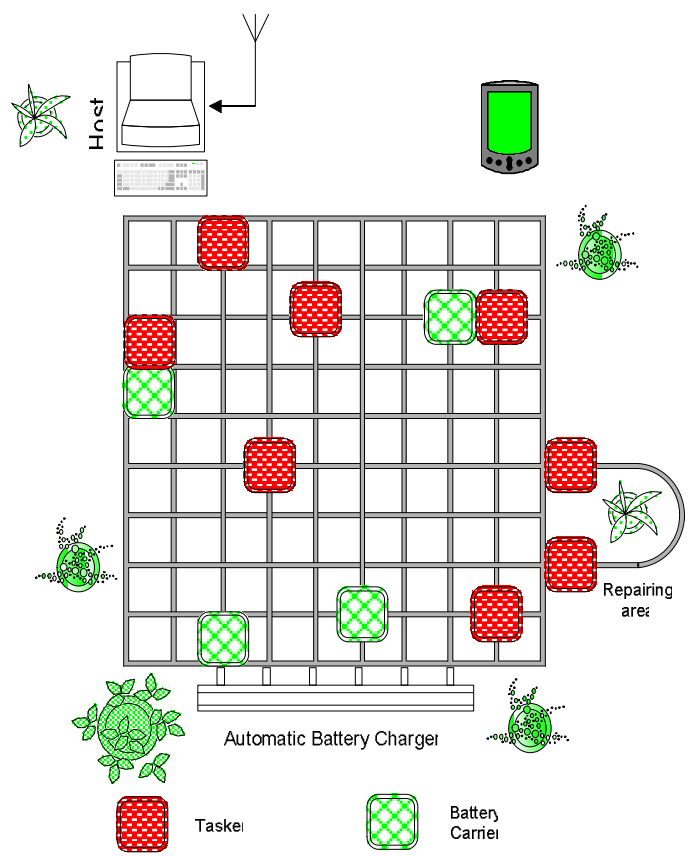

Fig.4. Experimental scenario for CISSbot battery exchange

In our system every robots is always set-up with the mission so there is not specified "tasker" robot as [15], where appears the model of master/slave in which some operates as worker without mission of carrying batteries and some works as battery "carrier", which only behaves as battery deliverer without other works. Therefore, in our system, the name "tasker" or "carrier" is temporally automatically assigned in short time, depending on probabilistic density function of total energy at a time in local vicinity. In fact, the radio communication range is much longer, the term "local" is correspondingly like "global" area, that is, every robot can communicate with others.

Thanks to infrared array of line tracking, compass and odometry, CISSbots can easily report and update its current position when following the white line to traverse on the field. In the setup, every robot is free to move randomly to consume its own energy. It sends out a radio signal of energy status and current position to host frequently, therefore the host can easily update current status of all robot moving. On the host, total energy of a robot is consequently synthesized and projected to corresponding rates of consumed energy tables, shown in figure 1, to give out suitable decision for such robots. Assume that energy of a robot "worker" approaches to cal for battery, it automatically sends signal of low power status to host and waiting for the reply. Simultaneously, the host will compute "worker"'s remaining energy capacity to refer to distance from current position to closest charging station. If the remain energy capacity can enable the robot move back to the charging station, the "worker" is guided to do. Otherwise, the "worker" is indicated to switch to standby mode to wait for rescuer The host will search to find suitable robots which is ready to exchange battery. If success, the host has to then find a non-collided path from "carrier" to "worker" and control the "carrier" to go "needed"'s current position to exchange battery. Otherwise, the host must add the "worker" to emergency mode. In the case, another robot will automatically to move to its position to full-haul it to the repairing station.

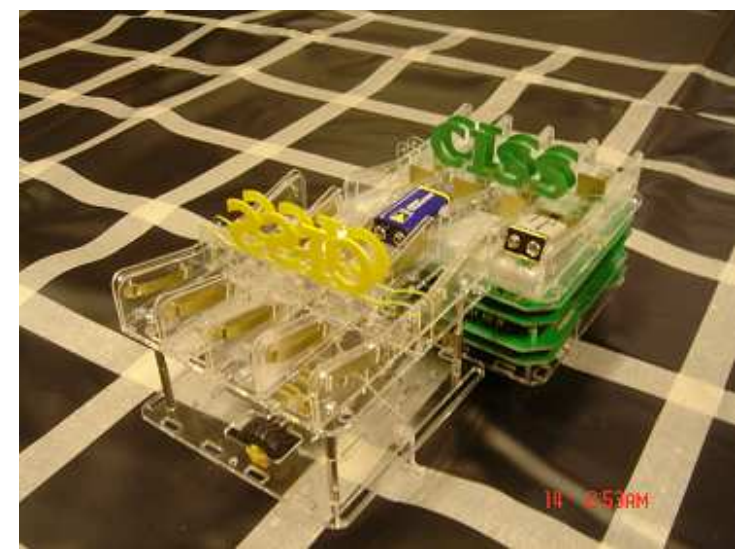

Fig.5. Two real CISSbots in state of exchanging battery

In process of battery exchange, two robots in battery exchange process can also communicate to arrange temporally meeting point, and simultaneously moving to the position. Then infrared local communication will conduct battery exchange by selecting the holder to be changed between robots: the usable battery on "tasker" is moved to empty holder of the "tasker" robot; discharged battery on the "tasker" is returned to the "carrier" if indicated. Further, using two essential variables of remaining energy and corresponding distance referred to battery station to build potential field in which robots with low energy and closer to charging station is nominated to become "carrier" to move back to the charging station to return batteries drain off and take full charged ones. Briefly, energy of the robotic system is harmonically distributed around the charging station as an attractive point. 


\section{CONCLUSION \& FUTURE WORK}

This paper presents a challenging trend of mobile robot towards truly autonomous mobile robot. The trend is a method of propagation of energy resources among mobile robots in order to keep group of mobile robot long-lived. The method is originally based on battery exchange between robots. To create rules of exchange, a general model of probabilistic distributed energy is established. However, the model will be able to be corrected and applied for specific case, depending on change of scenario. In the paper, we describe details of mechanical prototype, modular electronic and control architecture for CISSbot. The algorithm based on probabilistic energy distribution is specially emphasized in the paper. The experimental scenario shows that the system can be directly applied to industrial application in manufacture or deployed as military robots where robots need to be truly autonomous.

But, as not satisfied with current results, we have been upgrading advanced aspects for such robot to propose new application. In the future, we are concentrating on three problems of the system. First, we are focusing on problem of mother-children relation where heterogeneous mobile robot can work together in which CISSbot can behave as mobile charging stations for smaller robots. Secondly, we are setting up a chain of mobile robots using smart hooks. The chain is mainly expected to generate more stable chain of mobile robot than single robot to pass over all rough terrain. Thirdly, although it is the most difficult dimension we are towards to sensing techniques for self-localization of such mobile robot to remove grid mapping. All work will be modeled and deployed on our real robots with practical applications.

\section{REFERENCES}

[1] Chris Melhuish and Masao Kubo (2004). Collective Energy Distribution: Maintaining the Energy balance in Distributed Autonomous Robots, in the proceedings of 7 th International Symposium on Distributed Autonomous Robotic Systems, June 23-25, 2004 Toulouse, France,p261-70,2004.

[2] Breazeal. C, Towards Socible Robots, Robotics and Autonomous Systems 42 (2003) 167-175.

[3] Fong. T, Nourbakhsh. I, Dautenhahn. K, A survey of socially interactive robots, Robotics and Autonomous Systems 42 (2003) 143-166.

[4] Duffy, B.R., "Towards Social Intelligence in Autonomous Robotics: A Review", Robotics, Distance Learning and Intelligent Communication Systems 2001 (RODLICS 2001), Malta, September $1-6,2001$.

[5] Duffy, B.R., "Anthropomorphism and The Social Robot", Robot as Partner: An Exploration of Social Robots, 2002 IEEE/RSJ International Conference on Intelligent Robots and Systems, September 30 - October 4, EPFL, Switzerland, 2002.

[6] Duffy, B.R., "Social Embodiment in Autonomous Mobile Robotics", International Journal of Advanced Robotic Systems, 1 (3), 155-170, 2004

[7] Milo C. Silverman, Dan Nies, Boyoon Jung, and Gaurav S. Sukhatme. Staying alive: A docking station for autonomous robot recharging, in IEEE Intl. Conf. on Robotics and Automation, 2002

[8] A. Z. Seungjun Oh \& K. Taylor. Autonomous battery recharging for indoor mobile robots, in the proceedings of Australian Conference on Robotics and Automation (ACRA2000).
[9] Yongguo Mei, Yung-Hsiang Lu, C.S. George Lee and Y. Charlie Hu Energy-Efficient Motion Planning for Mobile Robots, International Conference on Robotics and Automation 2004.

[10] Yongguo Mei, Yung-Hsiang Lu, Y. Charlie Hu, C.S. George Lee. Deployment Strategy for Mobile Robots with Energy and Timing Constraints", International Conference on Robotics and Automation 2005.

[11] A. Barili, M.Ceresa, C Parisi. Energy-Saving Motion Control for An Autonomous Mobile Robot. In International Symposium on Industrial Electronics, pages 674-676, 1995

[12] Yasuhi Hada and Shin'ichi Yuta. Robust navigation and battery re-charging system for long term activity of autonomous mobile robots, in proceedings of the $9^{\text {th }}$ International conference on Advanced Robotics, October 1999, pp.297-302.

[13] F. Yamasaki, K. Hosoda and M.Asada. An Energy Consumption Based Control for Humannoid Walking, in IEEE/RSJ IROS, pages 2473-2477, 2002.

[14] NT. Ngo HH. Lund. Modern Ambient Intelligence Embodied in Distributed Modular Robotic Systems, In proceedings of the $3^{\text {rd }}$ International Conference in Computational Intelligence, Robotics, Autonomous Systems, Singapore, 2005

[15] Pawel Zebrowski, Richard Vaughan. Recharging Robot Teams: A Tanker Approach, International Conference on Advanced Robotics (ICAR'05), Seattle, Washington, July 18th-20th, 2005

[16] Ioannis Ieropoulos, Chris Melhuish and John Greenman (2004): 'Energetically Autonomous Robots', Proceedings of the 8th Intelligent Autonomous Systems Conference (IAS-8), Amsterdam, The Netherlands, pp 128-35.

[17] Kouronbov. K and Austin.D. Autonomous Recharging for Mobile Robotics, in proceedings of 2002 Australian Conference on Robotics and Automation, Auckland 27-29 Nov. 2002.

[18] Morten Winkler Jorgensen, Esben H. Ostergaard and Henrik Hautop Lund, "Modular ATRON: Modules for a self-reconfigurable robot", In proceedings of IEEE/RSJ International Conference on Robots and Systems, (IROS), Sendai, Japan, Pages 2068-2073, Sep 30-Oct 2, 2004.

[19] Mondada F., Pettinaro G.C., Guignard A., Kwee I., Floreano D., Deneubourg J.-L., Nolfi S., Gambardella L.M., Dorigo M., Swarm-Bot: a New Distributed Robotic Concept, Autonomous Robots, 17(2-3):193-221, 2004

[20] Mondada F., Bonani M., Magnenat S., Guignard A., Floreano D. In Frans Groen, Nancy Amato, Andrea Bonarini, Eiichi Yoshida and Ben Kröse editors, Physical connections and cooperation in swarm robotics, Proceedings of the 8th Conference on Intelligent Autonomous Systems (IAS8), Amsterdam, NL, March 10-14, 2004, pages 53-60. IOS Press. 